\title{
Metaheurísticas Aplicadas al Problema de Interdicción en Sistemas de Potencia
}

\author{
Juan J. Cortina ${ }^{(1)}$, Jesús M. López-Lezama ${ }^{(2) *}$ y Nicolás Muñoz-Galeano ${ }^{(2)}$ \\ (1) XM S.A. E.S.P. - Filial de ISA, Calle 12 Sur No. 18-168, Medellín - Colombia \\ (e-mail: jcortina@xm.com.co). \\ (2) Grupo de Investigación GIMEL, Departamento de Ingeniería Eléctrica, Facultad de Ingeniería, \\ Universidad de Antioquia, Calle 67 No.53-108, Medellín - Colombia (e-mail: jmaria.lopez@udea.edu.co; \\ nicolas.munoz@udea.edu.co).
}

${ }^{*}$ Autor a quien debe ser dirigida la correspondencia.

Recibido Jun. 20, 2017; Aceptado Sep. 5, 2017; Versión final Nov. 19, 2017, Publicado Abr. 2018

\begin{abstract}
Resumen
En este artículo se presenta una comparación de metaheurísticas aplicadas al problema de interdicción de sistemas de potencia. Este problema considera la interacción entre un agente disruptor y el operador de red. El agente disruptor tiene como objetivo causar el máximo daño al sistema, expresado en deslastre de carga. Este agente cuenta con recursos limitados y debe seleccionar la combinación de elementos a atacar que resulten en el mayor daño al sistema, anticipándose a la reacción del operador de red. Por otro lado, el operador de red debe minimizar el deslastre de carga mediante el redespacho de generación. El modelo de interdicción propuesto es no lineal, no conexo y multimodal. Para su solución se comparan tres metaheurísticas: Algoritmo Genético, GRASP y Búsqueda Local Iterada. Los resultados muestran que la Búsqueda Local Iterada se adapta de mejor manera al tipo de problema y permite obtener la mejor relación entre la calidad de las soluciones y el tiempo de ejecución.
\end{abstract}

\section{Metaheuristics Applied to the Power System Interdiction Problem}

\begin{abstract}
This paper presents a comparison of metaheuristics applied to the power system interdiction problem. This problem considers the interaction of a disruptive agent and a system operator. The disruptive agent aims at maximizing the damage of the power system, measured as load shedding. This agent has limited resources and must select the combination of elements to attack that result in the greatest damage to the system, anticipating the reaction of the system operator. On the other hand, the system operator must minimize the load shedding by redispatching generation resources. The interdiction model is nonlinear, nonconvex and multimodal. The solutions provided by three metaheuristic techniques are compared: Genetic Algorithm, GRASP and Iterated Local Search. Results show that the Iterated Local Search adapts better to the type of problem and allows obtaining the best rate between quality of solutions and computation time.
\end{abstract}

Keywords: genetic algorithms; local search; interdiction; vulnerability 


\section{INTRODUCCIÓN}

El problema de interdicción de sistemas de potencia fue propuesto por primera vez en (Salmeron et al., 2004). En contraste con los análisis típicos que consideran la salida fortuita de uno o dos elementos de la red (criterio $\mathrm{N}-1$ o N-2), Salmeron et al. (2004) proponen un análisis de vulnerabilidad introduciendo un nuevo concepto: la intencionalidad. En el modelo de interdicción se supone que existen dos agentes con funciones objetivo en conflicto. El agente disruptor busca estrategias para maximizar el deslastre de carga de la red, mientras el operador del sistema debe responder a cualquier ataque mediante el redespacho de generación para minimizar el deslastre de carga. El agente disruptor cuenta con recursos limitados y debe seleccionar el conjunto de elementos a atacar, anticipándose a la reacción del operador de red. La interacción de estos dos agentes se puede modelar de forma jerárquica, dando origen a un problema de programación binivel. Arroyo y Galiana (2005) presentan una generalización del problema de interdicción permitiendo definir funciones objetivo diferentes para ambos agentes. Arroyo y Fernández (2009) proponen la solución del problema de interdicción mediante un algoritmo genético y Arroyo (2010) propone modelos de mínima y máxima vulnerabilidad. El modelo de mínima vulnerabilidad busca encontrar el mínimo número de elementos que causen un deslastre de carga previamente especificado.

El modelo de máxima vulnerabilidad busca encontrar el máximo deslastre posible para un número dado de elementos a atacar. En ambos casos se utilizan modelos lineales de la red. Wang y Baldick (2014) presentan un modelo de interdicción que combina salidas de elementos en cascada e impactos de corto y mediano plazo. Los impactos de mediano plazo se incorporan mediante un modelo DC de la red y los impactos de corto plazo mediante un análisis de fallas en cascada. Sayyadipour et al. (2016) proponen un modelo de interdicción que determina no solo las líneas a ser atacadas, sino también el momento en el cual su ataque produce máximo daño bajo un horizonte de tiempo dado. En este caso se utiliza teoría de la dualidad para convertir el problema binivel en uno equivalente de un solo nivel y posteriormente se aplican técnicas de linealización al problema resultante para convertirlo en un problema de programación lineal entera mixta. Un elemento común de los trabajos mencionados anteriormente es que utilizan un modelo lineal de la red de transmisión (modelo DC).

Mantener niveles de confiabilidad y seguridad aceptables en los sistemas de potencia es uno de los principales retos de los operadores y planeadores de red. En este contexto se han propuesto gran variedad de estudios para detectar elementos críticos y aumentar la seguridad de los sistemas de potencia. Correa y Yusta (2014) estudian escenarios de riesgo basados en eventos que podrían desencadenar fallas en cascada en sistemas de potencia. Para ello utilizan técnicas basadas en teoría de grafos. Por otro lado, Alguacil et al., (2014) proponen un modelo de tres niveles para el problema de interdicción. En el nivel superior se ubica el planeador de la red que debe determinar los nuevos circuitos construir (refuerzos de red) teniendo en cuenta la interacción con los niveles medio e inferior donde se encuentran el agente disruptor y el operador de red, respectivamente. Finalmente, Salmeron y Wood (2015) incorporan el efecto de transformadores de respaldo y tiempos de reparación en el modelo de interdicción de sistemas de potencia.

El uso de modelos lineales para representar la red de transmisión permite convertir el problema binivel en un problema equivalente de un solo nivel. Para esto el nivel inferior de optimización es reemplazado por sus condiciones de optimalidad como se ilustra en (Arroyo, 2010) o se utiliza teoría de la dualidad y linealización como se ilustra en (Sayyadipour et al., 2016). Por otro lado, si el problema del nivel inferior es no lineal, el modelo no puede ser transformado en un equivalente de un solo nivel y se debe abordar en su forma original. Agudelo et al. (2014) abordan el problema de interdicción considerando un modelo no lineal de la red de transmisión (modelo AC). La ventaja de este tipo de modelos radica en que considera el efecto de las tensiones y de la potencia reactiva, lo que permite obtener soluciones más cercanas a la realidad. En (Agudelo et al., 2014) solo se consideran ataques a líneas y los autores utilizan un algoritmo híbrido que combina un algoritmo genético con búsqueda local para resolver el problema. López-Lezama et al. (2017) presentan un modelo de interdicción que también incorpora el modelo AC de la red y que además de ataques a líneas considera posibles ataques a generadores. Desde el punto de vista matemático el problema de interdicción, considerando el modelo AC de la red de transmisión es un problema no lineal, no convexo y multimodal. Las técnicas de programación matemática convencionales no resultan ser efectivas para abordar este tipo de problemas, por lo que se recurre al uso de técnicas metaheurísticas. El uso de este tipo de técnicas es común en la solución de problemas de programación binivel. Hong et al. (2014) y Hong et al. (2016) presentan algoritmos evolutivos para solucionar problemas de optimización binivel que solo involucran variables de decisión discretas. Kalyanmoy y Ankur (2010) presentan un método híbrido que combina una búsqueda local y un algoritmo evolutivo para solucionar problemas multi-objetivo de tipo binivel y Li et al. (2016) presentan un método basado en un algoritmo diferencial evolutivo para resolver problemas binivel mono-objetivo.

En este artículo se presenta una comparación de técnicas metaheurísticas para la solución del problema de interdicción considerando el modelo AC de la red de transmisión. Existe un gran número de metaheurísticas 
que pueden ser aplicadas al problema bajo estudio. En este caso se han seleccionado tres técnicas con diferentes filosofías de búsqueda. Las técnicas seleccionadas para comparación son: Algoritmo Genético (AG), Búsqueda Local Iterativa (BLI) y Procedimiento de Búsqueda Local Adaptativo Goloso (GRASP por sus siglas en inglés). El AG constituye un ejemplo de técnica poblacional que incorpora criterios evolutivos; la BLI es una técnica no poblacional que se basa en búsquedas locales sucesivas y el algoritmo GRASP es un método constructivo con búsqueda local. Se realizaron varias pruebas con los sistemas IEEE de 24 y 118 barras comparando la efectividad de las técnicas propuestas. Se pudo verificar que la técnica con mejor desempeño (en términos de calidad de soluciones y tiempo de cómputo) para solucionar el problema de interdicción es la BLI. Los resultados que arroja el modelo propuesto resultan de interés para el operador de red, pues se identifican los elementos cuya salida pueden causar el mayor daño al sistema. Con esta información el operador o el planeador de la red puede tomar decisiones sobre proteger ciertos elementos o reforzar la red.

\section{MODELO MATEMÁTICO}

Las ecuaciones (1) a (18) representan el modelo matemático de interdicción considerado el modelo AC de la red de transmisión.

$$
\max _{\delta^{B r}} \sum_{n} P_{D S_{n}} ; \quad \forall n \in N
$$

Sujeto a:

$$
\begin{aligned}
& \sum_{l}\left(1-\delta_{l}^{B r}\right) \leq M ; \quad \forall l \in B r \\
& \delta_{l}^{B r} \in\{0,1\} ; \quad \forall l \in B r \\
& \min _{\boldsymbol{x}} \sum_{g} c_{g} P_{g}^{G e n}+\sum_{n} c_{D S_{n}} P_{D S_{n}} \\
& \boldsymbol{x}=\left[\theta_{n}, V_{n}, P_{g}^{G e n}, Q_{g}^{G e n}, P_{l}^{B r}, Q_{l}^{B r}, P_{D S_{n}}, Q_{D S_{n}}\right]
\end{aligned}
$$

Sujeto a:

$$
\begin{aligned}
& \theta_{n}^{\text {min }} \leq \theta_{n} \leq \theta_{n}^{\max } ; \quad \forall n \in N \\
& V_{n}^{\text {min }} \leq V_{n} \leq V_{n}^{\text {max }} ; \quad \forall n \in N \\
& P_{g}^{\text {min }} \leq P_{g}^{G e n} \leq P_{g}^{\text {max }} ; \quad \forall g \in G e n \\
& Q_{g}^{\text {min }} \leq Q_{g}^{G e n} \leq Q_{g}^{\max } ; \quad \forall g \in G e n \\
& S_{l}^{\text {min }} \leq S_{l}^{B r} \leq S_{l}^{\max } ; \quad \forall l \in B r \\
& 0 \leq\left(P_{D S_{n}}\right) \leq P_{D_{n}} ; \quad \forall n \in N \\
& P_{n}=V_{n} \sum_{n} V_{m}\left[g_{m n} \cos \left(\theta_{m n}\right)+b_{m n} \sin \left(\theta_{m n}\right)\right] ; \quad \forall l \in B r, \quad \forall n \in N \\
& Q_{n}=V_{n} \sum_{n} V_{m}\left[g_{m n} \sin \left(\theta_{m n}\right)+b_{m n} \cos \left(\theta_{m n}\right)\right] ; \quad \forall l \in B r, \quad \forall n \in N \\
& \left(S_{l}^{B r}\right)^{2}=\left(P_{l}^{B r}\right)^{2}+\left(Q_{l}^{B r}\right)^{2} ; \quad \forall l \in B r \\
& P_{l}^{B r}=\delta_{l}^{B r} \cdot\left[g_{m n} V_{n}^{2}+g_{m n} V_{m} V_{n} \cos \left(\theta_{m n}\right)-b_{m n} V_{m} V_{n} \sin \left(\theta_{m n}\right)\right] ; \quad \forall l \in B r \\
& Q_{l}^{B r}=\delta_{l}^{B r} \cdot\left[-b_{m n} V_{n}^{2}+b_{m n} V_{m} V_{n} \cos \left(\theta_{m n}\right)-b_{m n} V_{m} V_{n} \sin \left(\theta_{m n}\right)\right] ; \quad \forall l \in B r \\
& P_{g}^{G e n}-P_{D_{n}}+P_{D S_{n}}=P_{n} ; \quad \forall n \in N
\end{aligned}
$$




$$
\begin{aligned}
& Q_{g}^{G e n}-Q_{D_{n}}+Q_{D S_{n}}=Q_{n} ; \quad \forall n \in N \\
& \theta_{\text {ref }}=0
\end{aligned}
$$

En estas ecuaciones la notación usada es la siguiente:

Índices y conjuntos

$\begin{array}{ll}m, n & \text { Índices que denotan barras del sistema. } \\ l, \mathrm{~g} & \text { Índice de ramas y generadores, respectivamente. } \\ \mathrm{Br} & \text { Conjunto de ramas (líneas y transformadores). } \\ \mathrm{N} & \text { Conjunto de barras del sistema de potencia. } \\ \mathrm{Gen} & \text { Conjunto de generadores del sistema de potencia. }\end{array}$

Parámetros:

M Recursos disponibles totales para realizar un ataque, expresado en unidades monetarias.

$c_{g} \quad$ Precios de oferta de la generación, expresado en unidades monetarias por megavatio.

$c_{D S_{n}} \quad$ Costo de racionamiento de carga activa en la barra $n$, expresado en unidades monetarias por megavatio.

$P_{D_{n}}, Q_{D_{n}} \quad$ Demanda de potencia activa y reactiva en la barra $n$, respectivamente.

$P_{g}^{\max }, P_{g}^{\min }$ Límites máximo y mínimo de generación de potencia activa en la barra $n$, respectivamente.

$Q_{g}^{\max }, Q_{g}^{\min }$ Límites máximo y mínimo de generación de potencia reactiva en la barra $n$, respectivamente.

$P_{l}^{\max }, P_{l}^{\min } \quad$ Límites máximo y mínimo de potencia activa por la línea $l$, respectivamente.

$Q_{l}^{\text {max }}, Q_{l}^{\text {min }}$ Límites máximo y mínimo de potencia reactiva por la línea $l$, respectivamente.

$S_{l}^{\max }, S_{l}^{\min }$ Límites máximo y mínimo de potencia aparente por la línea $l$, respectivamente.

$\theta_{n}^{\max }, \theta_{n}^{\min }$ Límites máximo y mínimo de ángulo de fase en la barra $n$, respectivamente.

$V_{n}^{\text {max }}, V_{n}^{\text {min }}$ Límites máximo y mínimo de magnitud de tensión en la barra $n$, respectivamente.

$g_{m n} \quad$ Conductancia de la línea $l$ que interconecta las barras $m$ y $n$.

$b_{m n} \quad$ Susceptancia de la línea $l$ que interconecta las barras $m$ y $n$.

$\theta_{m n} \quad$ Diferencia angular de las barras $m$ y $n$.

Variables:

$\delta_{l}^{B r} \quad$ Vector de interdicción de ramas (líneas o transformadores).

$P_{D S_{n}}, Q_{D S_{n}}$ Deslastre de potencia activa y reactiva en la barra $n$, respectivamente.

$P_{l}^{L i n}, Q_{l}^{L i n} \quad$ Flujo de potencia activa y reactiva por la línea $l$, respectivamente.

$P_{g}^{G e n}, Q_{g}^{G e n}$ Generación de potencia activa del generador $g$, respectivamente.

$P_{n}, Q_{n} \quad$ Inyecciones de potencia activa y reactiva en la barra $n$, respectivamente.

$S_{l}^{L i n} \quad$ Flujo de potencia aparente por la línea $l$.

$\theta_{n}, V_{n} \quad$ Ángulo de fase y magnitud de tensión en la barra $n$, respectivamente

Las ecuaciones (1) a (3) corresponden al problema de optimización del nivel superior. La ecuación (1) representa el objetivo del agente disruptor que consiste en maximizar la demanda deslastrada. La ecuación (2) indica los límites en recursos destructivos y la ecuaciones (3) la naturaleza binaria de las variables de 
decisión. En este caso un valor "1" en el vector $\delta_{l}^{B r}$ indica que el elemento (línea o transformador) no ha sido atacado y se encuentra en operación, por otro lado un valor "0" indica que el elemento respectivo fue atacado.

La función objetivo del operador de red está dada por la ecuación (4), la cual consiste en minimizar el costo de racionamiento y de atención de la demanda. El primer término indica el costo de atender la demanda del sistema y el segundo el costo de racionamiento. Dada una decisión del nivel superior, el operador de red debe redespachar sus recursos de generación cumpliendo con las restricciones de red. Las ecuaciones (5) a (10) modelan las restricciones de desigualdad en las que los superíndices min y max se refieren a los límites de las variables. Los límites en ángulos de fase y magnitud de las tensiones en todos los nodos están dados por las ecuaciones (5) y (6), respectivamente. Las ecuaciones (7) y (8) representan los límites de generación de potencia activa y reactiva, respectivamente. La ecuación (9) representa los límites en el flujo de potencia aparente por las líneas de la red. Finalmente, la ecuación (10) considera el hecho de que el deslastre de carga en una barra no puede superar la demanda de la respectiva barra.

Las restricciones de igualdad están dadas por las ecuaciones (11) a (18). Las expresiones matemáticas de las inyecciones nodales de potencia activa y reactiva están dadas por las ecuaciones (11) y (12), respectivamente. La ecuación (13) indica las componentes activa y reactiva del flujo de potencia aparente en las líneas de la red. Las ecuaciones (14) y (15) definen los flujos de potencia activa y reactiva entre dos nodos. Las ecuaciones (16) y (17) representan los balances nodales de potencia activa y reactiva, respectivamente. Finalmente, la ecuación (18) define la referencia angular del sistema.

\section{METAHEURÍSTICAS IMPLEMENTADAS}

El problema descrito en la sección anterior se abordó mediante diferentes técnicas de optimización metaheurística. Esto con el objetivo de comparar su desempeño en función de la calidad de las soluciones obtenidas y el tiempo de cómputo. Se seleccionaron tres de las técnicas más conocidas en la literatura. Una técnica poblacional (AG) y dos técnicas basadas en búsqueda local (GRASP e ILS).

\section{Representación de soluciones}

Un candidato de solución es representado mediante el vector binario $\delta_{l}^{B r}$. Este vector, a su vez, puede ser representado por un vector de menor longitud de variables enteras que indiquen el elemento bajo ataque. Ambas representaciones reciben el nombre de Vector de Interdicción (VI). En la Fig. 1 se ilustra un ejemplo de un VI en sus representaciones binaria y entera. El sistema de la Fig. 1 cuenta con 13 ramas. Un plan de ataque para eliminar cuatro elementos (L1, L5, L10 y L12) se puede representar mediante un vector binario de 13 posiciones donde se indiquen los estados operativos de los elementos (1 si está activo y 0 si es atacado); o alternativamente mediante un vector de solo cuatro posiciones (con variables enteras) que indiquen el número del elemento bajo ataque.

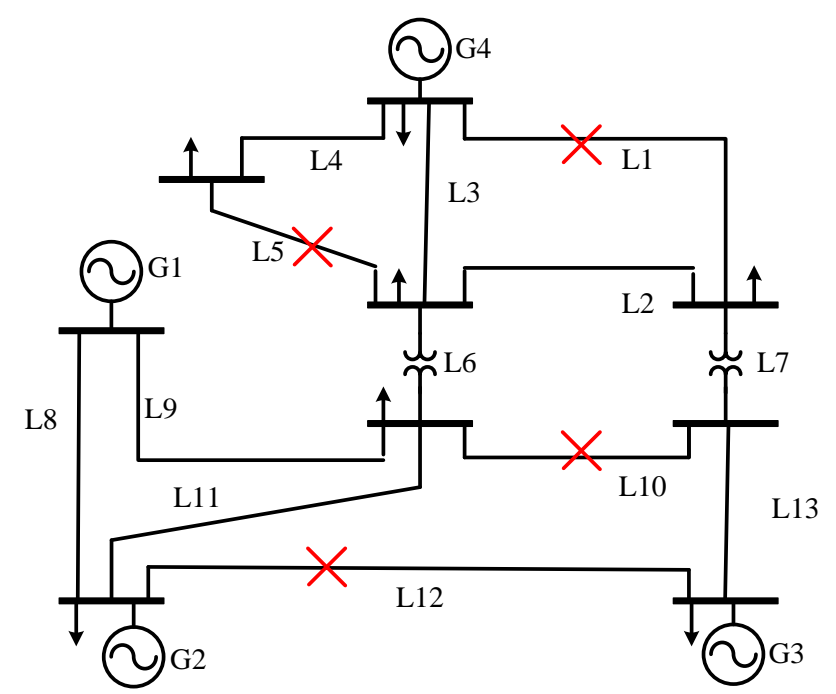

a)

\begin{tabular}{|c|c|c|c|c|c|c|c|c|c|c|c|c|} 
L1 & L2 & L3 & L4 & L5 & L6 & L7 & L8 & L9 & L10 & L11 & L12 & L13 \\
\hline 0 & 1 & 1 & 1 & 0 & 1 & 1 & 1 & 1 & 0 & 1 & 0 & 1 \\
\hline
\end{tabular}

b) \begin{tabular}{|c|c|c|c|} 
L1 & L5 & \multicolumn{1}{c}{ L10 } & L12 \\
\hline 1 & 5 & 10 & 12 \\
\hline
\end{tabular}

Fig. 1: llustración de un Vector de Interdicción: a) Representación binaria b) Representación entera 


\section{Búsqueda Local Iterativa (BLI)}

La BLI es un procedimiento que a partir de una solución inicial busca un óptimo local. Una vez se encuentra dicho óptimo local, la solución es perturbada para ubicarse en un punto diferente del espacio de búsqueda y hacer nuevamente otra búsqueda local (ver Fig. 2). Este procedimiento se repite un número especificado de iteraciones o hasta que se haya conseguido una solución satisfactoria. En la Fig. 3 se ilustra el diagrama de flujo de la BLI. En este caso, para garantizar soluciones de alta calidad el VI inicial es construido siguiendo el algoritmo heurístico propuesto por López-Lezama et al. (2017). Este algoritmo consiste en eliminar un elemento a la vez verificando que no se supere el límite de recursos con los que cuenta el agente atacante (ver ecuación (2)). La búsqueda local se realiza mediante la variación aleatoria y simultánea de dos componentes del VI en su representación entera. El procedimiento se hace un número determinado de veces y se selecciona el mejor individuo encontrado. La perturbación de la BLI se realiza iniciando nuevamente el procedimiento desde otro punto del espacio de búsqueda. Esto implica encontrar un nuevo VI de forma similar a como se encontró la solución inicial.

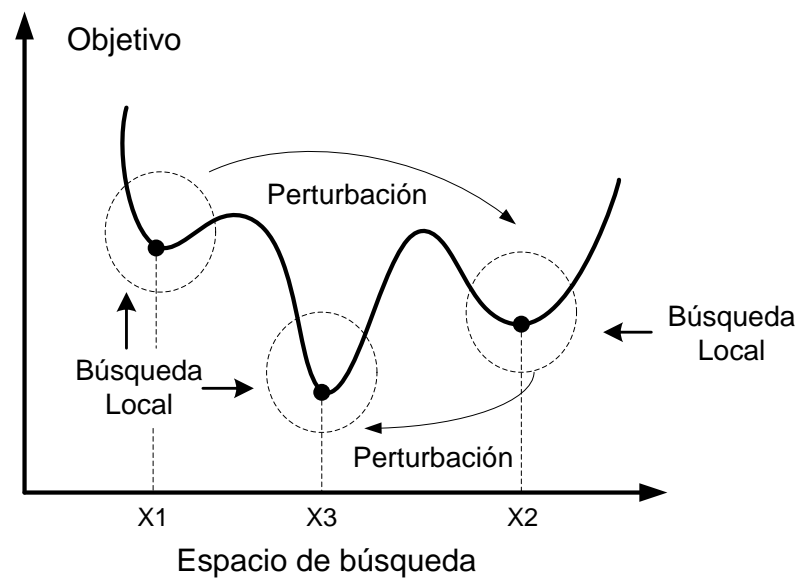

Fig.2: Principio de funcionamiento de la BLI

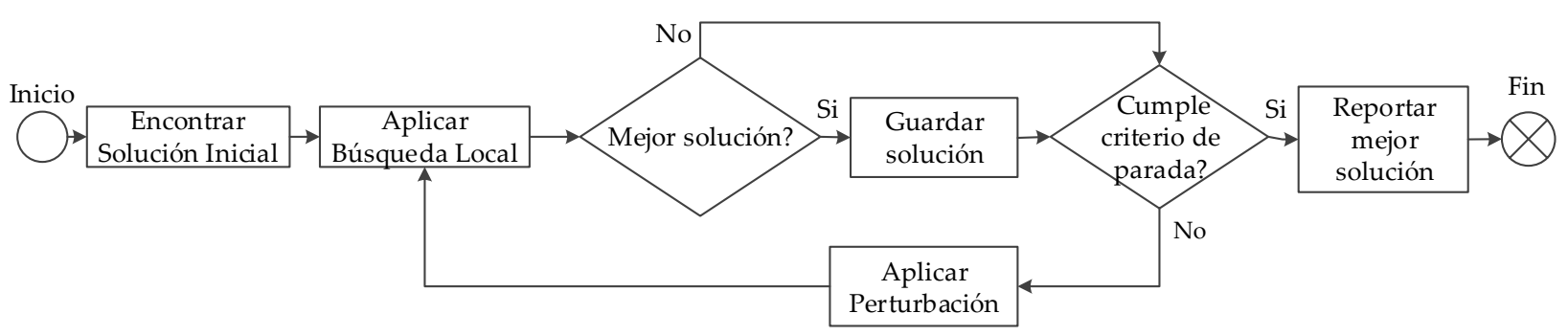

Fig.3: Diagrama de flujo de la BLI

\section{Algoritmo Genético (AG)}

Un AG es una metaheurística poblacional que se inspira en el proceso de adaptación de las especies. Dada una población inicial, esta es sometida a las etapas de selección, recombinación y mutación de forma sucesiva con el objetivo de encontrar mejores individuos. En el AG implementado, los candidatos de solución (vectores de interdicción) representan individuos de una población.

El AG parte de un conjunto de $\mathrm{N}$ individuos diferentes (siendo $\mathrm{N}$ el tamaño de la población, el cual se mantiene constante). La creación de los individuos se hace de forma tal que todos sean factibles en costos; es decir, que no violen la restricción dada por la ecuación (2). Una vez se tiene una población inicial, se evalúa la función objetivo como se indica en la ecuación (1). A partir de la población inicial se genera una nueva población. Para ello se selecciona un conjunto de individuos de la población actual mediante torneos. Un torneo consiste en tomar un subconjunto $\mathrm{k}$ del conjunto de $\mathrm{N}$ individuos y seleccionar el mejor.

Los individuos con mejor función objetivo se seleccionan como "padres"; los cuales se recombinan para generar nuevos individuos. En la Fig. 4 se ilustra el proceso de selección. La recombinación se hace punto a punto usando la codificación entera de los VI. Si en la recombinación se genera una infactibilidad en costos, los individuos son reparados disminuyendo el número de elementos atacados. Después de la recombinación 
los nuevos individuos pasan a la etapa de mutación. Esta etapa consiste en hacer pequeños cambios a los individuos con cierta probabilidad. La mutación consiste en atacar elementos vecinos a los propuestos en el plan de ataque inicial. Para mantener la población constante se eliminan los elementos con peor función objetivo (reducción de la población). El proceso continúa hasta completar un número dado de generaciones. En la Fig. 5 se ilustra el diagrama de flujo del AG. Una descripción más detallada de la aplicación de los AG en el problema de interdicción puede ser consultada en (Cortina 2016).

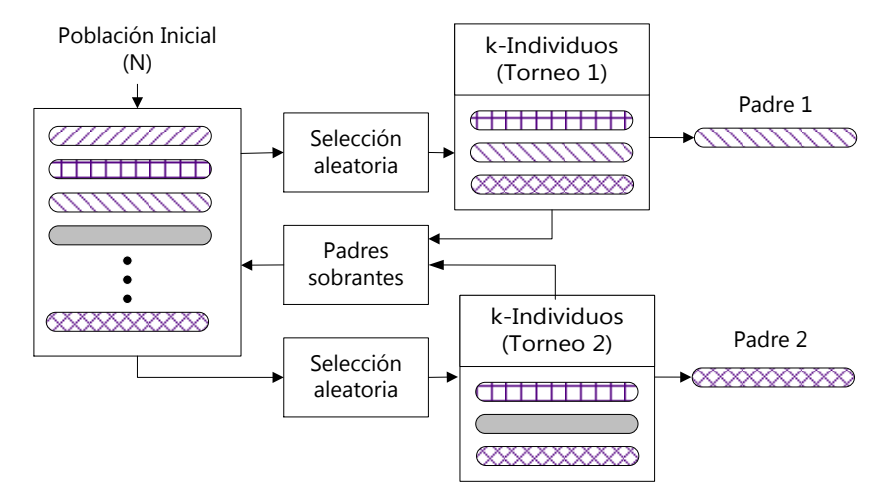

Fig. 4: Ilustración del proceso de selección del AG.

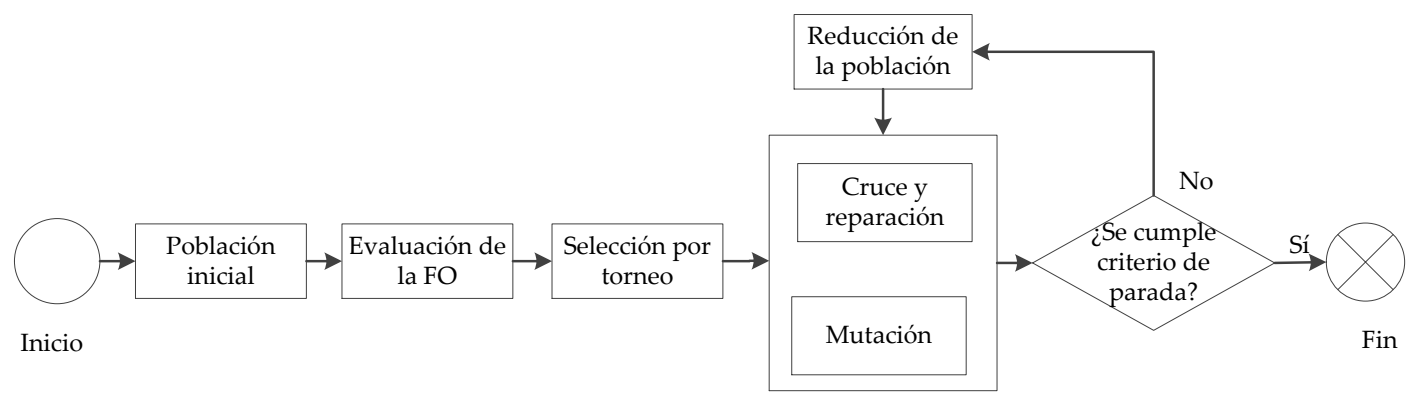

Fig. 5: Diagrama de flujo del AG.

\section{Búsqueda Adaptativa GRASP}

El método GRASP consiste en crear una solución inicial y después efectuar una búsqueda local para mejorar la calidad de la solución. La solución inicial del método GRASP se obtiene mediante el mismo algoritmo constructivo usado en la BLI. Una vez se tiene la solución inicial la búsqueda local se hace mediante dos movimientos. El primer movimiento está definido por la variación aleatoria y simultánea de dos componentes del VI. La variación es aceptada si el VI resultante es factible en costos (cumple con la restricción (2)) y su función objetivo (FO) es mejor que la de la solución inicial. El procedimiento finaliza cuando se cumple el criterio de máxima o primera mejora. El criterio de máxima mejora, consiste en encontrar una mejor solución dentro de un número dado de iteraciones y el criterio de primera mejora, consiste en encontrar la primera mejor solución. La Fig. 6 representa el primer movimiento de la búsqueda local, el cual define el primer vecindario de búsqueda. Una vez se ha cumplido alguno de los criterios descritos, se aplica el segundo movimiento, el cual está definido por la variación aleatoria de cada componente del VI; aplicando el criterio de primera o máxima mejora. El segundo movimiento determina el segundo vecindario de la búsqueda local y es representado en la Fig. 7 .

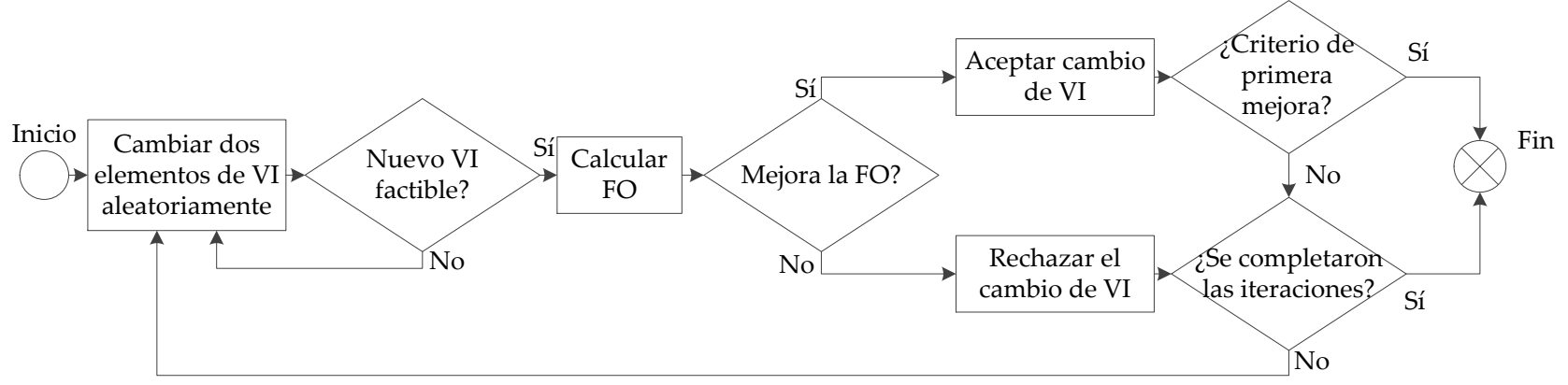

Fig. 6: Primer movimiento de búsqueda local para el método GRASP. 


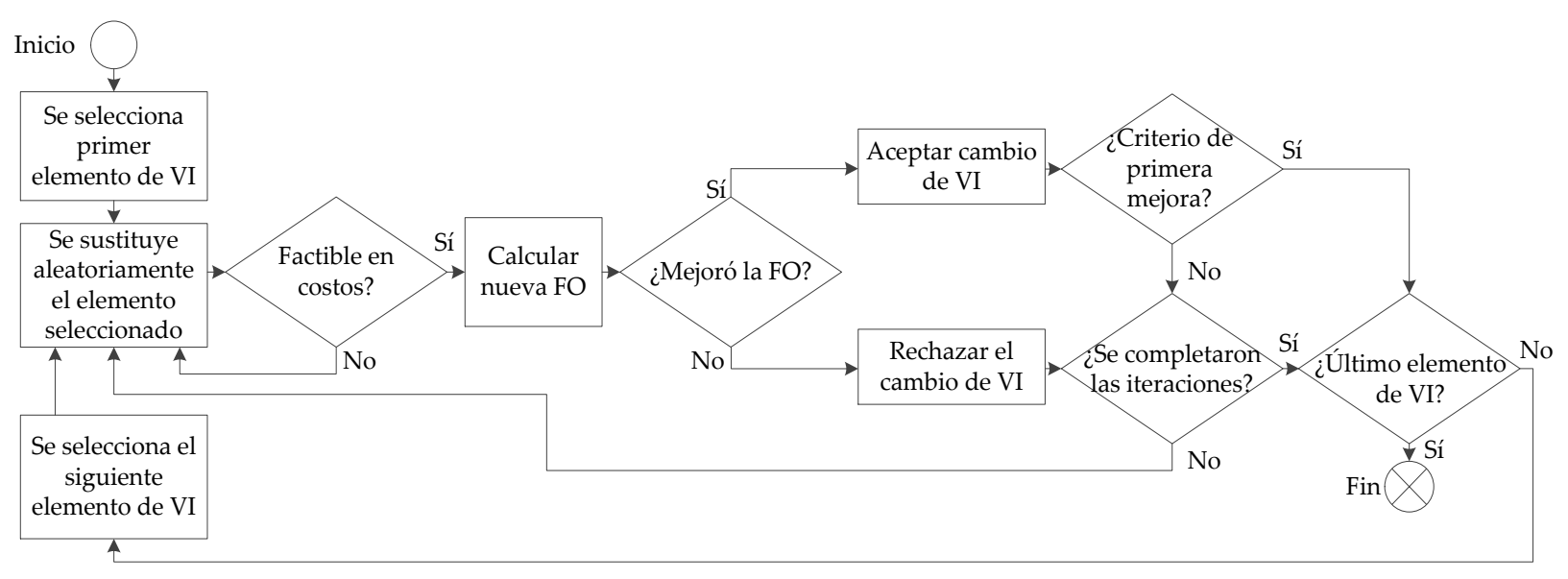

Fig. 7: Segundo movimiento de búsqueda local para el método GRASP

\section{PRUEBAS Y RESULTADOS}

Para comparar la efectividad de los métodos bajo estudio se utilizaron los sistemas de prueba IEEE de 24 y 118 barras. Los datos de red de estos sistemas pueden ser consultados en (Grigg et al., 1999) y (University of Washington, 2016), respectivamente. Todas las pruebas se realizaron en un computador portátil con 6.0GB de memoria RAM y un procesador core-i5 de 4 núcleos de $1.6 \mathrm{GHz}$. Para calcular la respuesta del operador de red (el redespacho de generación ante ataques) se utilizó el software de optimización MATPOWER (Zimmerman et al., 2011). Por simplicidad se asumió que atacar cualquier rama del sistema (línea 0 transformador) tiene el mismo costo. Para modelar el deslastre de carga se utilizaron generadores ficticios en las barras de carga. El costo de racionamiento para ambos casos se asumió igual a 5 veces el precio de oferta del generador más costoso (US\$200/MW). Por otro lado, cuando se generan islas se crea una barra de referencia en cada una de éstas para garantizar convergencia del flujo de carga. Finalmente, las soluciones infactibles son descartadas del proceso de solución.

\section{Resultados con el sistema IEEE 24 barras}

Este sistema cuenta con 17 barras de carga, 11 generadores y 38 ramas. Las pruebas se realizaron para un día de invierno a las 18 horas, con una demanda total de $2850 \mathrm{MW}$ y una capacidad de generación instalada de $3405 \mathrm{MW}$. Por simplicidad, se considera $P_{g}^{\min }=0 \mathrm{MW}$ para todos los generadores. La calibración de los parámetros para cada una de las técnicas bajo estudio se ilustra a continuación. Para encontrar los ajustes de las metaheurísticas y con el fin de garantizar la reproducibilidad de los resultados, se aprovecharon dos hechos en común de las tres técnicas: 1) Emplean el mismo heurístico constructivo para determinar un tamaño de población inicial o conjunto de candidatos de solución inicial y 2) Su criterio de parada está dado por un número máximo de iteraciones (perturbaciones en el caso de la BLI y generaciones en el caso del $A G$ ).

Por ello, se realizaron varias simulaciones con el fin de identificar el número de individuos o candidatos de solución y el número máximo de iteraciones que optimizan la ejecución de las técnicas. Previamente, se realizaron otros ensayos donde se observó mejor desempeño de las metaheurísticas con los parámetros adicionales que se listan a continuación, los cuales fueron fijados al momento de las variaciones del Número de individuos - Iteraciones. Para la BLI se consideraron 6 iteraciones en la búsqueda local para variar un elemento dentro de un candidato de solución y criterio de máxima mejora para esta búsqueda local. Para el GRASP se usó una lista restringida de 3 candidatos para tratar de variar cada elemento dentro de un candidato de solución y criterio de máxima mejora para la búsqueda local. Finalmente, para el AG se realizó cruce por un solo punto, $10 \%$ de probabilidad de mutación de un individuo resultante luego de la recombinación y elitismo como criterio de reducción para elegir la población de la siguiente generación.

A continuación se ilustran resultados de pruebas de calibración de los algoritmos donde se asumió que el agente disruptor contaba con un límite de recursos destructivos de 6 unidades monetarias $(M=6)$, siendo el costo de ataque de una rama del sistema una unidad monetaria. Las Fig. 8, Fig. 9 y Fig. 10 muestran los resultados obtenidos variando el número de los candidatos de solución en 20,50 y 100, con un número máximo de iteraciones de 20, 50 y 100. Los factores medidos fueron el tiempo promedio de ejecución de las metaheurísticas, los máximos valores de deslastre de carga alcanzados en cada ejecución y la dispersión de los deslastres obtenidos entre una ejecución y otra. En este caso se realizaron 5 ejecuciones de cada algoritmo para cada combinación de parámetros Número de individuos - Iteraciones. 


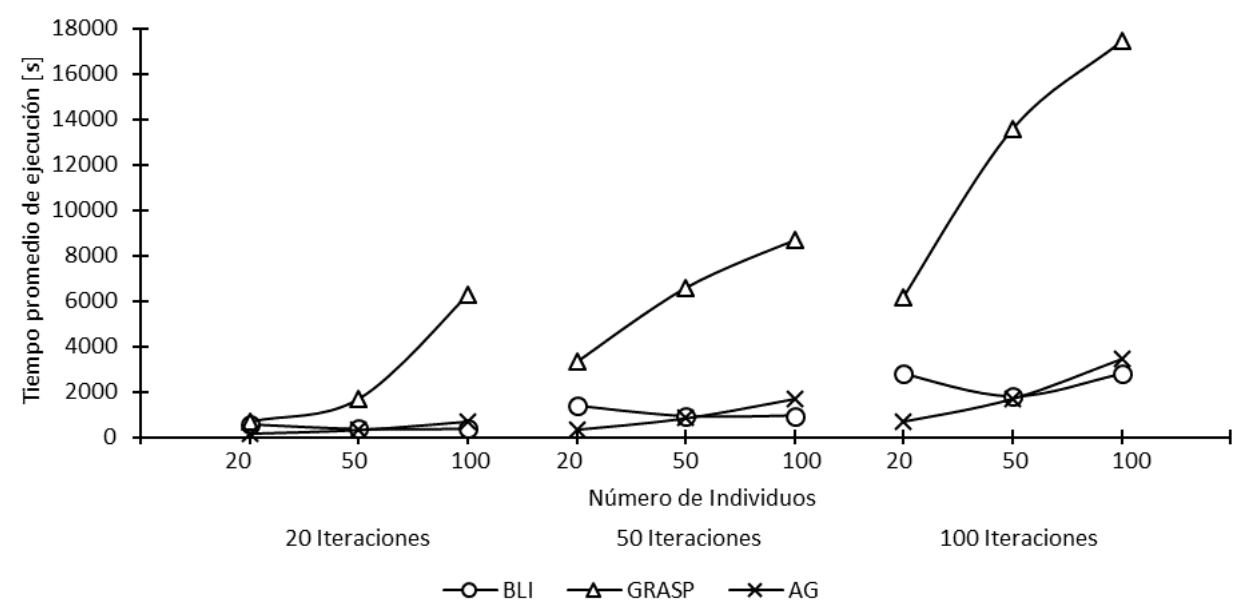

Fig. 8: Desempeño en tiempos de ejecución variando la relación Número de Individuos - Iteraciones

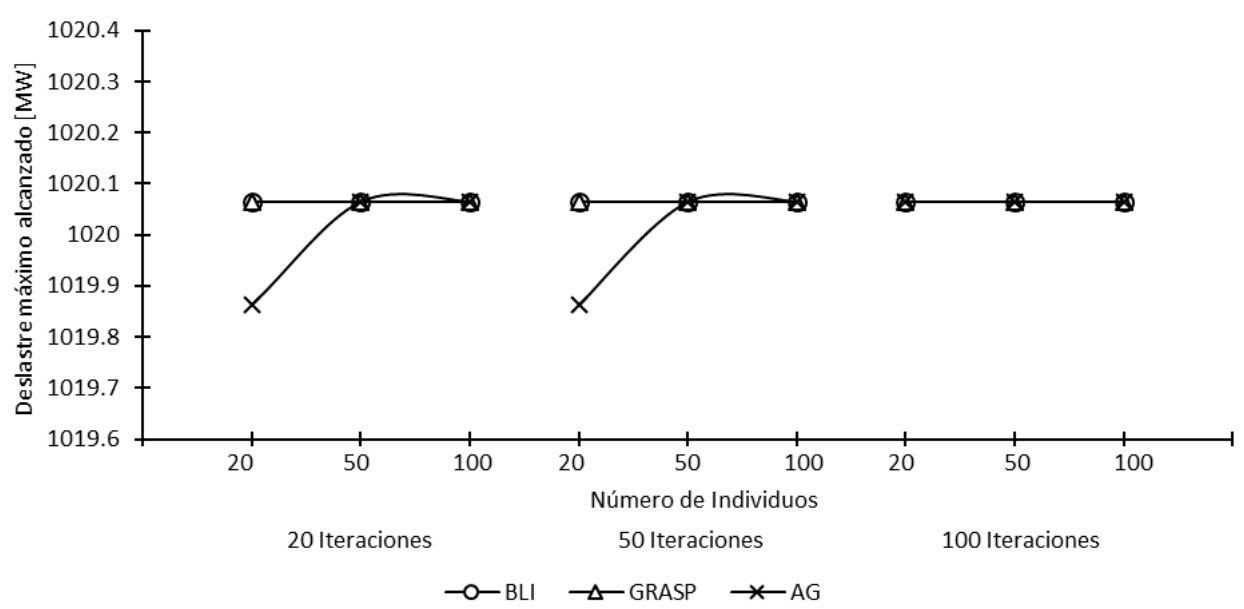

Fig. 9: Calidad de las soluciones variando la relación Número de Individuos - Iteraciones

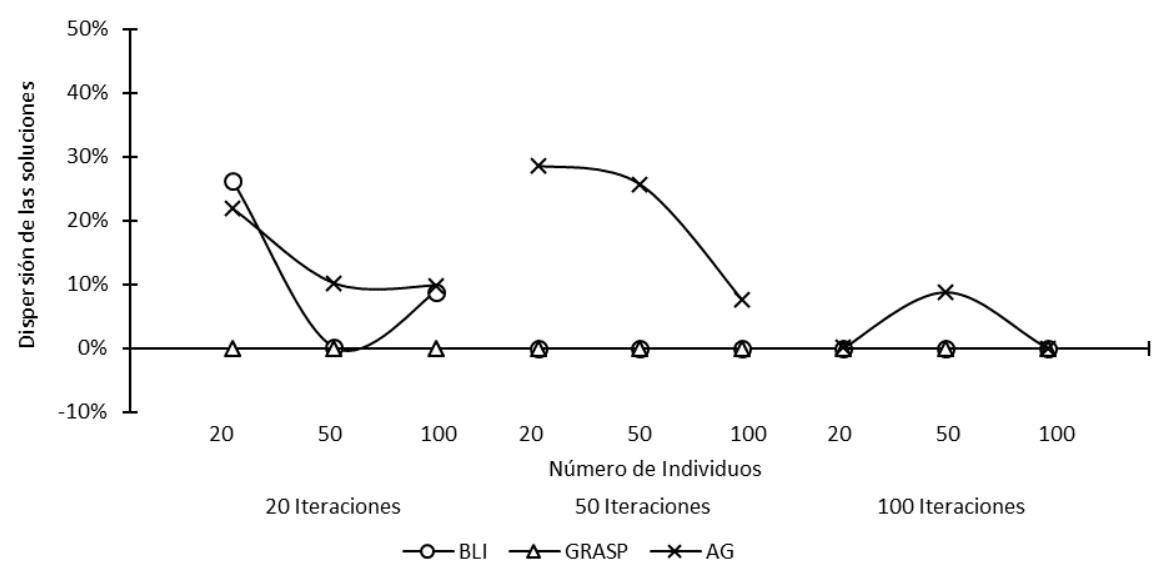

Fig. 10: Dispersión [\%] entre las soluciones variando la relación Número de Individuos - Iteraciones

De los resultados se aprecia que a medida que se incrementa el número de iteraciones, el tiempo de cómputo de las metaheurística es mayor, tal como se evidencia en la Fig. 8; no obstante, la metaheurística GRASP presenta los mayores tiempos de ejecución a medida que el número de individuos y el número de iteraciones aumenta. Por sus características constructivas, las técnicas GRASP y AG ven afectado su desempeño en tiempo de ejecución, Sin embargo, se advierte que esto no ocurre con la BLI. Para esta técnica, al aumentar el número de individuos, manteniendo constante el número iteraciones, no necesariamente se aumentan los tiempos de ejecución. Esto se debe a que el conjunto de soluciones iniciales de la BLI, le permite encontrar una buena solución semilla que mejora con la búsqueda local. Partiendo de una buena solución semilla, se logra que la BLI llegue a mejores resultados en menor tiempo. 
En la Fig. 9 se observa que para todas las combinaciones de Número de Individuos - Iteraciones los deslastres alcanzados con la BLI y el GRASP fueron el máximo valor posible para $M=6$. Este resultado puede ser corroborado en (Agudelo et al., 2014). Estos resultados corresponden con los reportados en (Arroyo 2010), en cuanto a los elementos atacados. En el AG se evidencia que, para un tamaño de población de 20 individuos, la calidad de las soluciones no es muy buena cuando se tienen 20 y 50 generaciones. Sin embargo, al aumentar el número de generaciones, indistintamente del tamaño de la población, siempre se obtienen los mejores resultados. Esto último va en detrimento del desempeño de la técnica, pues requiere más tiempo de cómputo para su ejecución.

En la Fig. 10 se ilustra el grado de dispersión de las soluciones encontradas para las 5 ejecuciones de cada combinación de Número de Individuos - Iteraciones. El grado de dispersión entre soluciones fue calculado a partir del coeficiente de variación, definido como el cociente entre la desviación estándar sobre el promedio de las soluciones obtenidas. Se observa que el GRASP, en todos los casos, fue consistente con las soluciones encontradas ( $0 \%$ de dispersión), es decir 100\% de reproducibilidad, aunque a costa de mayores tiempos de ejecución (ver Fig. 8). La BLI, con 20 individuos y 20 iteraciones, en al menos 3 de 5 ejecuciones obtiene el máximo deslastre de carga; sin embargo, cuando se incrementa el número de iteraciones, se logra el $100 \%$ de reproducibilidad. En particular, a partir de 50 iteraciones la dispersión de los resultados es $0 \%$ para 20, 50 o 100 individuos. El AG presenta la mayor dispersión en las técnicas analizadas. Para 20 individuos, se consigue $0 \%$ de dispersión en las soluciones encontradas cuando se tienen muchas generaciones. Con 50 individuos siempre hubo dispersión en las soluciones encontradas y con 100 individuos y 100 generaciones se logra el $100 \%$ de reproducibilidad, aunque con mayor tiempo de ejecución.

De acuerdo con lo anterior, se parametrizaron las metaheurísticas en cuanto al número de individuos y el máximo de iteraciones buscando un equilibrio entre tiempos de respuesta y calidad de las soluciones. Para la BLI se usaron 100 candidatos de solución inicial y 20 perturbaciones. Para el GRASP 20 soluciones iniciales y 20 iteraciones en la búsqueda local. Finalmente, para el AG se consideraron 100 individuos en la población inicial y 50 generaciones.

Con los parámetros fijados anteriormente y los valores que se seleccionaron para el Número de individuos e Iteraciones, se obtuvieron los resultados mostrados en la Tabla 1a y Tabla 1b. Estos resultados son comparados con los obtenidos con el modelo de interdicción DC reportados en (Arroyo, 2010). Las ramas se representan con los números de las barras entre las que se encuentran conectadas, separados por un guion.

Tabla 1a: Mejores VI obtenidos con diferentes técnicas de búsqueda (sistema IEEE 24 barras)

\begin{tabular}{|c|c|c|c|}
\hline Método & Mejor VI con $M=2$ & $\begin{array}{c}\text { Deslastre } \\
\text { en MW }\end{array}$ & $\begin{array}{c}\text { Tiempo } \\
{[\mathrm{s}]}\end{array}$ \\
\hline BLI & $11-14,14-16$ & 194 & 44.81 \\
\hline GRASP & $11-14,14-16$ & 194 & 318.43 \\
\hline AG & $11-14,14-16$ & 194 & 92.78 \\
\hline Arroyo (2010) & $11-14,14-16$ & 194 & 291.01 \\
\hline
\end{tabular}

\begin{tabular}{|c|c|c|c|}
\hline Método & Mejor VI con $M=4$ & $\begin{array}{c}\text { Deslastre } \\
\text { en MW }\end{array}$ & $\begin{array}{c}\text { Tiempo } \\
{[\mathrm{s}]}\end{array}$ \\
\hline BLI & $12-23,13-23,14-16,15-24$ & 526.8567 & 90.76 \\
\hline GRASP & $12-23,13-23,14-16,15-24$ & 526.8567 & 516.171 \\
\hline AG & $2-4,16-19,20-23,20-23$ & 309 & 186.87 \\
\hline Arroyo (2010) & $3-24,12-23,13-23,14-16$ & 516 & 15981.94 \\
\hline
\end{tabular}

\begin{tabular}{|c|c|c|c|}
\hline Método & Mejor VI con $M=6$ & $\begin{array}{c}\text { Deslastre } \\
\text { en MW }\end{array}$ & $\begin{array}{c}\text { Tiempo } \\
{[\mathrm{s}]}\end{array}$ \\
\hline BLI & $3-24,7-8,9-12,10-12,11-13,14-16$ & 1020.065 & 383.12 \\
\hline GRASP & $3-24,7-8,9-12,10-12,11-13,14-16$ & 1020.065 & 681.81 \\
\hline AG & $7-8,9-12,10-12,11-13,14-16,15-24$ & 1020.065 & 340.77 \\
\hline Arroyo (2010) & $3-24,7-8,9-12,10-12,11-13,14-16$ & 1017 & 3575.68 \\
\hline
\end{tabular}


Tabla 1b: Mejores VI obtenidos con diferentes técnicas de búsqueda (sistema IEEE 24 barras)

\begin{tabular}{|c|c|c|c|}
\hline Método & Mejor VI con $M=8$ & $\begin{array}{c}\text { Deslastre } \\
\text { en MW }\end{array}$ & $\begin{array}{c}\text { Tiempo } \\
{[\mathrm{s}]}\end{array}$ \\
\hline BLI & $9-12,10-12,11-13,15-21,15-21,16-17$, \\
$20-23,20-23$ & 1205.6 & 267.54 \\
\hline GRASP & $7-8,9-12, \begin{array}{c}10-12,11-13,14-16,15-16, \\
15-21,15-21\end{array}$ & 1122.1 & 933.15 \\
\hline AG & $1-3,1-5,3-24,7-8,9-12,10-12,11-13,14-16$ & 1023.5055 & 483.28 \\
\hline Arroyo $(2010)$ & $9-12,10-12,11-13,15-21,15-21,16-17$, \\
$20-23,20-23$ & 1198 & 1171.13 \\
\hline
\end{tabular}

\begin{tabular}{|c|c|c|c|}
\hline Método & Mejor VI con $M=10$ & $\begin{array}{c}\text { Deslastre } \\
\text { en MW }\end{array}$ & $\begin{array}{c}\text { Tiempo } \\
{[\mathrm{s}]}\end{array}$ \\
\hline BLI & $\begin{array}{c}1-5,7-8,9-12,10-12,11-13,15-21,15-21, \\
16-17,20-23,20-23\end{array}$ & 1377.8176 & 382.20 \\
\hline GRASP & $\begin{array}{c}1-5,2-4,7-8,9-12,10-12,11-13,14-16, \\
15-16,15-21,15-21\end{array}$ & 1126.9 & 1121.95 \\
\hline AG & $\begin{array}{c}1-2,1-5,2-4,2-6,7-8,9-12,10-12,11-13, \\
14-16-24\end{array}$ & 1116.0359 & 491.53 \\
\hline Arroyo (2010) & $\begin{array}{r}7-8,9-12,10-12,11-13,15-21,15-21,16-17, \\
20-23,20-23\end{array}$ & 1373 & 42.78 \\
\hline
\end{tabular}

\begin{tabular}{|c|c|c|c|}
\hline Método & Mejor VI con $M=12$ & $\begin{array}{c}\text { Deslastre } \\
\text { en MW }\end{array}$ & $\begin{array}{c}\text { Tiempo } \\
\text { [s] }\end{array}$ \\
\hline BLI & $\begin{array}{c}1-2,1-3,1-5,7-8,9-12,10-12,11-13,15-21, \\
15-21,16-17,20-23,20-23\end{array}$ & 1459.1735 & 490.04 \\
\hline GRASP & $\begin{array}{c}1-2,1-3,1-5,7-8,9-12,10-12,11-13,14-16, \\
15-24,16-19,20-23,20-23\end{array}$ & 1412.1 & 1307.67 \\
\hline AG & $\begin{array}{c}1-2,2-4,2-6,3-24,7-8,11-13,12-13,12-23, \\
14-16,16-19,20-23,20-23\end{array}$ & 1422.3163 & 499.95 \\
\hline Arroyo (2010) & $\begin{array}{c}1-2,2-4,2-6,7-8,11-13,12-13,12-23, \\
15-21,15-21,16-17,20-23,20-23\end{array}$ & 1468 & 588.63 \\
\hline
\end{tabular}

\begin{tabular}{|c|c|c|c|}
\hline Método & Mejor $V I$ con $M=14$ & $\begin{array}{c}\text { Deslastre } \\
\text { en MW }\end{array}$ & $\begin{array}{c}\text { Tiempo } \\
{[\mathrm{s}]}\end{array}$ \\
\hline BLI & $\begin{array}{c}1-3,1-5,2-4,2-6,7-8,9-12,10-11,10-12, \\
11-13,15-16,15-24,16-17,20-23,20-23\end{array}$ & 1460.9531 & 649.71 \\
\hline GRASP & $\begin{array}{c}1-3,1-5,2-4,2-6,7-8,10-11,11-13,12-13 \\
12-23,15-21,15-21,16-17,20-23,20-23\end{array}$ & 1552.7 & 1431.75 \\
\hline AG & $\begin{array}{c}1-3,1-5,2-6,4-9,7-8,10-11,11-13,12-13, \\
12-23,15-21,15-21,16-17,20-23,20-23\end{array}$ & 1478.7843 & 473.35 \\
\hline Arroyo (2010) & $\begin{array}{c}1-3,1-5,2-4,2-6,7-8,11-13,12-13,12-23, \\
14-16,15-21,15-21,16-17,20-23,20-23\end{array}$ & 1552 & 15.35 \\
\hline
\end{tabular}

\begin{tabular}{|c|c|c|c|}
\hline Método & Mejor $V I$ con $M=16$ & $\begin{array}{c}\text { Deslastre } \\
\text { en MW }\end{array}$ & $\begin{array}{c}\text { Tiempo } \\
{[\mathrm{s}]}\end{array}$ \\
\hline BLI & $\begin{array}{c}1-2,2-4,2-6,4-9,5-10,7-8,10-11,10-12 \\
11-13,12-13,12-23,14-16,15-24,16-19,20- \\
23,20-23\end{array}$ & 1445.9736 & 799.78 \\
\hline GRASP & $\begin{array}{c}1-3,1-5,2-4,2-6,7-8,9-12,10-11,10-12 \\
11-13,14-16,15-16,15-21,15-21,16-19,20- \\
23,20-23\end{array}$ & 1611.78 & 1613.25 \\
\hline$A G$ & $\begin{array}{c}1-3,1-5,2-4,2-6,7-8,9-11,10-11,10-12 \\
11-13,12-13,12-23,15-21,15-21,16-17,20- \\
23,20-23\end{array}$ & 1565.6438 & 473.54 \\
\hline Arroyo (2010) & $\begin{array}{c}-3,1-5,2-4,2-6,7-8,9-12,10-12,11-13 \\
14-16,15-16,15-21,15-21,16-19 \\
20-23,20-23\end{array}$ & 1607 & 20.92 \\
\hline
\end{tabular}


En la Tabla 1a y Tabla 1b se confirma que las técnicas de búsqueda diseñadas entregan resultados de alta calidad. En la mayoría de los casos se encontró la solución óptima, tomando como referencia la reportada en (Arroyo 2010), la cual corresponde a un método exacto para el problema de interdicción DC. Salvo para $M=$ 12 no fue posible reproducir el óptimo global; sin embargo, los resultados alcanzados por las distintas técnicas son de alta calidad y cercanos entre sí. La BLI alcanzó un deslastre de 1459.17 MW, el GRASP 1412.1 MW y el AG $1422.31 \mathrm{MW}$. Estos resultados difieren en menos de un 4\% con respecto a $1469.4 \mathrm{MW}$ que corresponde a la solución calculada con el modelo AC para las líneas reportadas en (Arroyo, 2010).

Se puede observar que para $M=2,4,6,8$ y 10, la BLI siempre obtuvo las soluciones óptimas. Para $M=14$ y 16, el GRASP obtuvo las soluciones óptimas. Solo en pocos casos, para pocas ramas atacadas, el AG fue capaz de obtener las soluciones óptimas, aunque en los casos con muchas ramas atacadas es la técnica más rápida en la entrega de buenas soluciones. La técnica menos efectiva para muchas ramas acatadas fue la BLI, pues sus resultados fueron inferiores a los del GRASP y a los del AG. En promedio, los tiempos de respuesta del GRASP son tres veces los tiempos empleados por la BLI y entre dos y tres veces los tiempos de cómputo empleados por el AG.

En la Fig. 11 se ilustran los mejores planes de ataque para el sistema IEEE de 24 barras $\operatorname{con} M=4,6$ y 8 . Se puede observar que los ataques buscan aislar las zonas donde se concentra la generación del sistema de las zonas donde se concentra la carga. En el caso de cuatro ramas atacadas, al inhabilitar los circuitos 14-16 y 15-24, en conjunto con los circuitos 12-23 y 13-23, queda represada la generación de las barras 18, 21, 22 y 23. Estas cuatro barras de generación constituyen cerca del $52 \%$ del total de la capacidad instalada y con este plan de ataque se produce un deslastre de 526.86 MW (18.49\% del total de la carga). Este resultado fue posible obtenerlo además de la BLI, con el GRASP, pero esta última técnica tardó más de 5 veces el tiempo empleado por la BLI. Con seis ramas atacadas ocurre una separación de las áreas eléctricas aguas arriba y abajo de los trasformadores de potencia.

El área superior queda en exceso de generación y el área inferior en déficit; pues en esta área se concentra la mayor parte de la carga del sistema (13332 MW de carga con solo $684 \mathrm{MW}$ de generación). Adicionalmente, el generado de la barra 7 (300 MW) queda aislado del sistema con el ataque al circuito 7-8, entregando únicamente la generación que corresponde a la demanda de la propia barra (125 MW). En todo el sistema, con seis ramas atacadas se logra un deslastre de $1020.065 \mathrm{MW}$ (35.79\% del total de la carga). Este resultado se corresponde con el hallado en (Arroyo 2010) y fue posible reproducirlo con las tres metaheurísticas. Para un ataque simultáneo a 8 ramas, se aíslan las barras 7,13 y 23 del resto del sistema, dejando en estas tres barras $1551 \mathrm{MW}$ de generación alimentando solo $390 \mathrm{MW}$ de carga de las barras 7 y 13 . El resto del sistema queda en déficit de generación frente a la carga que debe ser abastecida (1854 MW de capacidad instalada y $2460 \mathrm{MW}$ de carga). Adicionalmente, al inhabilitarse el doble circuito 15-21, toda la energía proveniente de la generación de las barras 18, 21 y 22, queda restringida por la transferencia máxima permitida a través del circuito 16-17, lo que restringe mucho más la generación y genera mayor deslastre. Para 8 ramas atacadas se obtiene un deslastre total de $1205.6 \mathrm{MW}$ (42.30\% del total de la carga del sistema). En este caso, solo fue posible reproducir el óptimo global con la BLI.

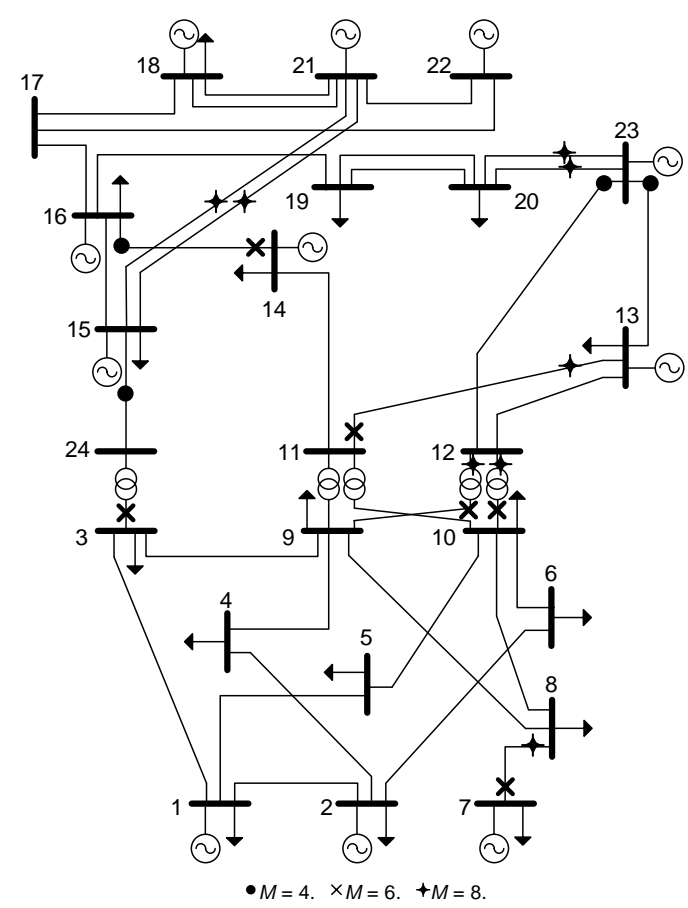

Fig. 11: Mejores planes de ataque hallados con las distintas técnicas de búsqueda - Sistema IEEE 24 barras 


\section{Resultados con el sistema IEEE 118 barras}

Este sistema cuenta con 19 generadores, 35 condensadores sincrónicos, 177 líneas, 9 transformadores y 91 cargas; con una capacidad de generación instalada de $9962.2 \mathrm{MW}$ y una carga de $4242 \mathrm{MW}$. Al igual que en el caso de 24 barras, se asumió por simplicidad que la potencia mínima que pueden entregar los generadores es cero $\left(P_{g}^{\min }=0 \mathrm{MW}\right)$. En este caso, siguiendo un procedimiento similar al descrito en el caso anterior, las metaheurísticas fueron calibradas para garantizar la reproducibilidad de los resultados. Para la BLI se utilizaron 100 individuos como candidatos de solución inicial, 30 perturbaciones, 30 iteraciones en la búsqueda local y criterio de máxima mejora para esta búsqueda local. Para el GRASP se consideraron 50 candidatos de solución inicial, 50 iteraciones, lista restringida de 3 candidatos para la búsqueda local y criterio de máxima mejora. Finalmente, para el AG se utilizaron 100 individuos en la población inicial, 100 generaciones, cruce por un solo punto, $10 \%$ de probabilidad de mutación y elitismo como criterio de reducción de las poblaciones. En la Tabla $2 a$ y Tabla $2 b$ se ilustran los resultados de los mejores planes de ataque obtenidos con las tres metaheurísticas para diferentes valores de $M$.

Tabla 2a: Mejores VI obtenidos con diferentes técnicas de búsqueda (sistema IEEE 118 barras)

\begin{tabular}{|c|c|c|c|}
\hline Método & Mejor $V I$ con $M=2$ & $\begin{array}{l}\text { Deslastre } \\
\text { en MW }\end{array}$ & $\begin{array}{l}\text { Tiempo } \\
{[\mathrm{s}]}\end{array}$ \\
\hline BLI & $77-78,79-80$ & 110 & 206.03 \\
\hline GRASP & $77-78,79-80$ & 110 & 1673.53 \\
\hline$A G$ & $71-72,68-116$ & 84 & 308.24 \\
\hline Método & Mejor $V I$ con $M=4$ & $\begin{array}{l}\text { Deslastre } \\
\text { en MW }\end{array}$ & $\begin{array}{l}\text { Tiempo } \\
{[\mathrm{s}]}\end{array}$ \\
\hline $\mathrm{BLI}$ & $77-78,79-80,68-116,12-117$ & 214 & 851.68 \\
\hline GRASP & $65-66,68-116,77-78,79-80$ & 194 & 9717.10 \\
\hline$A G$ & $19-20,22-23,68-116,12-117$ & 146 & 2786.42 \\
\hline Método & Mejor $V I \operatorname{con} M=6$ & $\begin{array}{l}\text { Deslastre } \\
\text { en MW }\end{array}$ & $\begin{array}{l}\text { Tiempo } \\
\text { [s] }\end{array}$ \\
\hline BLI & $19-20,22-23,77-78,79-80,68-116,12-117$ & 256 & 1082.87 \\
\hline GRASP & $11-12,19-20,22-23,77-78,79-80,68-116$ & 236 & 4157 \\
\hline$A G$ & $26-25,59-60,77-78,79-80,17-113,68-116$ & 194.0001 & 1426.51 \\
\hline Método & Mejor $V I$ con $M=8$ & $\begin{array}{l}\text { Deslastre } \\
\text { en MW }\end{array}$ & $\begin{array}{l}\text { Tiempo } \\
\text { [s] }\end{array}$ \\
\hline BLI & $\begin{array}{c}\text { 19-20, 22-23, 51-52, 53-54, 77-78, 79-80, } \\
68-116,12-117\end{array}$ & 297 & 3194.95 \\
\hline GRASP & $\begin{array}{c}19-20,21-22,35-36,77-78,79-80,68-116 \\
75-118,76-118\end{array}$ & 259 & 17368 \\
\hline$A G$ & $\begin{array}{c}38-65,47-69,76-77,77-78,79-80,80-98,68- \\
116,75-118\end{array}$ & 195.1495 & 2202.43 \\
\hline Método & Mejor $V I$ con $M=10$ & $\begin{array}{l}\text { Deslastre } \\
\text { en MW }\end{array}$ & $\begin{array}{l}\text { Tiempo } \\
\text { [s] }\end{array}$ \\
\hline BLI & $\begin{array}{c}27-28,29-31,47-49,24-72,77-78,79-80 \\
85-88,88-89,68-116,12-117\end{array}$ & 303 & 2016.09 \\
\hline GRASP & $\begin{array}{c}\text { 68-116, 106-107, 94-100, 56-69, 56-58, } \\
53-54,51-52,49-51,31-32,23-24\end{array}$ & 324 & 5360.05 \\
\hline$A G$ & $\begin{array}{c}\text { 37-39, 39-40, 51-52, 53-54, 59-60, 77-78, } \\
79-80,68-116,75-118,76-118\end{array}$ & 295.0001 & 1356.67 \\
\hline Método & Mejor $V I$ con $M=12$ & $\begin{array}{l}\text { Deslastre } \\
\text { en MW }\end{array}$ & $\begin{array}{l}\text { Tiempo } \\
\text { [s] }\end{array}$ \\
\hline BLI & $\begin{array}{c}12-16 ; 16-17 ; 77-78 ; 79-80 ; 85-88 ; 88-89 \\
94-95,80-98,95-96,98-100,68-116,12-117\end{array}$ & 363 & 7838.93 \\
\hline GRASP & $\begin{array}{c}19-20,21-22,40-41,41-42,77-78,79-80 \\
100-101,101-102,27-115,114-115,68-116 \\
12-117\end{array}$ & 327 & 27654.59 \\
\hline$A G$ & $\begin{array}{c}\text { 15-17, 23-24, 28-29, 24-72, 75-77, 77-78, } \\
\text { 79-80, 89-90, 100-101, 92-102, 105-106, } \\
68-116\end{array}$ & 221 & 2133 \\
\hline
\end{tabular}


Tabla 2b: Mejores VI obtenidos con diferentes técnicas de búsqueda (sistema IEEE 118 barras)

\begin{tabular}{|c|c|c|c|}
\hline Método & Mejor $V I$ con $M=14$ & $\begin{array}{l}\text { Deslastre } \\
\text { en MW }\end{array}$ & $\begin{array}{c}\text { Tiempo } \\
\text { [s] }\end{array}$ \\
\hline BLI & $\begin{array}{c}\text { 12-16, 19-20, 22-23, 40-41, 41-42, 49-50, } \\
49-51,53-54,56-57,51-58,77-78,79-80 \\
68-116,12-117\end{array}$ & 380.0001 & 4826 \\
\hline GRASP & $\begin{array}{c}\text { 19-20, 22-23, 50-57, 61-62, 77-78, 79-80, } \\
85-88,88-89,105-108,109-110,27-115 \\
114-115,68-116,12-117\end{array}$ & 336.0001 & 10156.26 \\
\hline$A G$ & $\begin{array}{c}12-16,19-20,20-21,22-23,27-28,29-31 \\
31-32,34-36,62-67,66-67,68-116,12-117 \\
75-118,76-118\end{array}$ & 248 & 1374.18 \\
\hline
\end{tabular}

\begin{tabular}{|c|c|c|c|}
\hline Método & Mejor $V I$ con $M=16$ & $\begin{array}{l}\text { Deslastre } \\
\text { en MW }\end{array}$ & $\begin{array}{c}\text { Tiempo } \\
\text { [s] }\end{array}$ \\
\hline BLI & $\begin{array}{c}\text { 8-9, 8-5, 15-17, 17-18, 25-27, 19-34, } \\
34-36,34-37,37-39,39-40,44-45,50-57 \\
38-65,65-68,69-70,68-116\end{array}$ & 1261.873 & 5330.29 \\
\hline GRASP & $\begin{array}{c}\text { 8-5, 17-18, 18-19, 15-19, 22-23, 26-25, } \\
15-33,47-49,38-65,71-72,77-78,79-80 \\
92-93,94-95,94-100,68-116\end{array}$ & 1778.9 & 11777.21 \\
\hline$A G$ & $\begin{array}{c}77-78,49-51,53-54,22-23,68-116,19-20 \\
70-71,47-49,79-80,11-13,13-15,62-67 \\
\quad 66-67,105-107,33-37,56-58\end{array}$ & 368 & 1233.97 \\
\hline
\end{tabular}

En la Tabla 2a y Tabla 2b se puede observar que, en la mayoría de los casos, los mejores resultados en cuanto a la calidad de la solución son alcanzados por la BLI, salvo para $M=10$ y $M=16$, donde el GRASP tuvo un mejor comportamiento, pero a costa de mayores tiempos de ejecución. En todos los ensayos variando $M$, siempre la BLI y el GRASP obtuvieron valores cercanos (las diferencias en los deslastres alcanzados variaban a lo sumo en $1.04 \%$, excepto para $M=16$ donde el GRASP obtuvo un deslastre de $1778.9 \mathrm{MW}(41.94 \%$ del total de la carga del sistema) y la BLI $1261.87 \mathrm{MW}$ (29.75\% del total de la carga). En ninguno de los ensayos el AG pudo alcanzar deslastres superiores a la BLI y al GRASP y para valores de $M$ mayores a 10, quedó atrapado en óptimos locales cuya calidad en solución era muy inferior a los encontrados con las otras dos técnicas.

La Fig. 12 muestra el sistema de prueba IEEE de 118 barras y los mejores planes obtenidos para $M=4, M=8$ y $M=12$. En el caso de 4 ramas atacadas de manera simultánea se observa que los circuitos 68-116 y 12-117 son radiales y al ser atacados, las cargas conectadas en las barras 116 y 117, quedan aisladas del sistema. En la barra 116 existe una generación con capacidad máxima instalada de $100 \mathrm{MW}$, pero su carga es de $184 \mathrm{MW}$, lo cual deja un deslastre en esta barra de $84 \mathrm{MW}$. La barra 117 no cuenta con generación, por lo cual los $20 \mathrm{MW}$ de carga en esta barra son deslastrados completamente. Los otros dos circuitos, 77-78 y 79-80, que complementan las cuatro ramas atacadas, aíslan también del sistema las barras 78 y 79 , las cuales no cuentan con generación, deslastrando $71 \mathrm{MW}$ y $39 \mathrm{MW}$, respectivamente. En total, para cuatro ramas atacadas (según la respuestas encontrada con la BLI) se produce un deslastre de $214 \mathrm{MW}$ (5.04\% del total de la carga). El mismo conjunto de líneas atacadas se repite en los planes de ataque hallados para $M=8$ y $M=12$; lo que muestra que estos cuatro circuitos son críticos para la red (en particular, los circuitos 68-116 y 12-117, que son radiales).

Con $M=8$ se combinan, además de los cuatro circuitos atacados para el caso $M=4$, los circuitos $19-20$ y 22 23, que aíslan del sistema las barras 20, 21 y 22 (18 MW, $14 \mathrm{MW}$ y $10 \mathrm{MW}$, respectivamente), junto con los circuitos 51-52 y 53-54 que deslastran las cargas de las barras 52 y 53 (18 MW y $23 \mathrm{MW}$, respectivamente). En total, con $M=8$, el mejor plan de ataque obtenido con las metaheurísticas, alcanzó un deslastre de $297 \mathrm{MW}(7 \%$ del total de la carga del sistema), en el cual fue deslastrada el 100\% de la carga en 7 de las 8 barras afectadas, ya que estas no cuentan con generación local. Para $M=12$, se produce deslastre en las barras ya mencionadas para $M=4$ y se combinan otros ataques a los circuitos 12-16 y 16-17 (25 MW deslastrados en la barra 16), 8588 y 88-89 (77 MW en la barra 88), 94-95 y 95-96 (42 MW en la barra 95) y los circuitos 80-98 y 98-100, que aíslan el $100 \%$ de la carga en la barra 98 (34 MW).

En las pruebas con el sistema IEEE de 118 barras se aprecia la tendencia de ataques en secciones radiales de la red o en ramas que atienden barras de carga donde no existe generación localizada. Este resultado era lo esperado porque, aunque el sistema tiene una capacidad de generación muy superior a la demanda, cuenta con gran cantidad de tramos radiales y 40 de las 54 barras de carga no cuentan con generación localizada. 


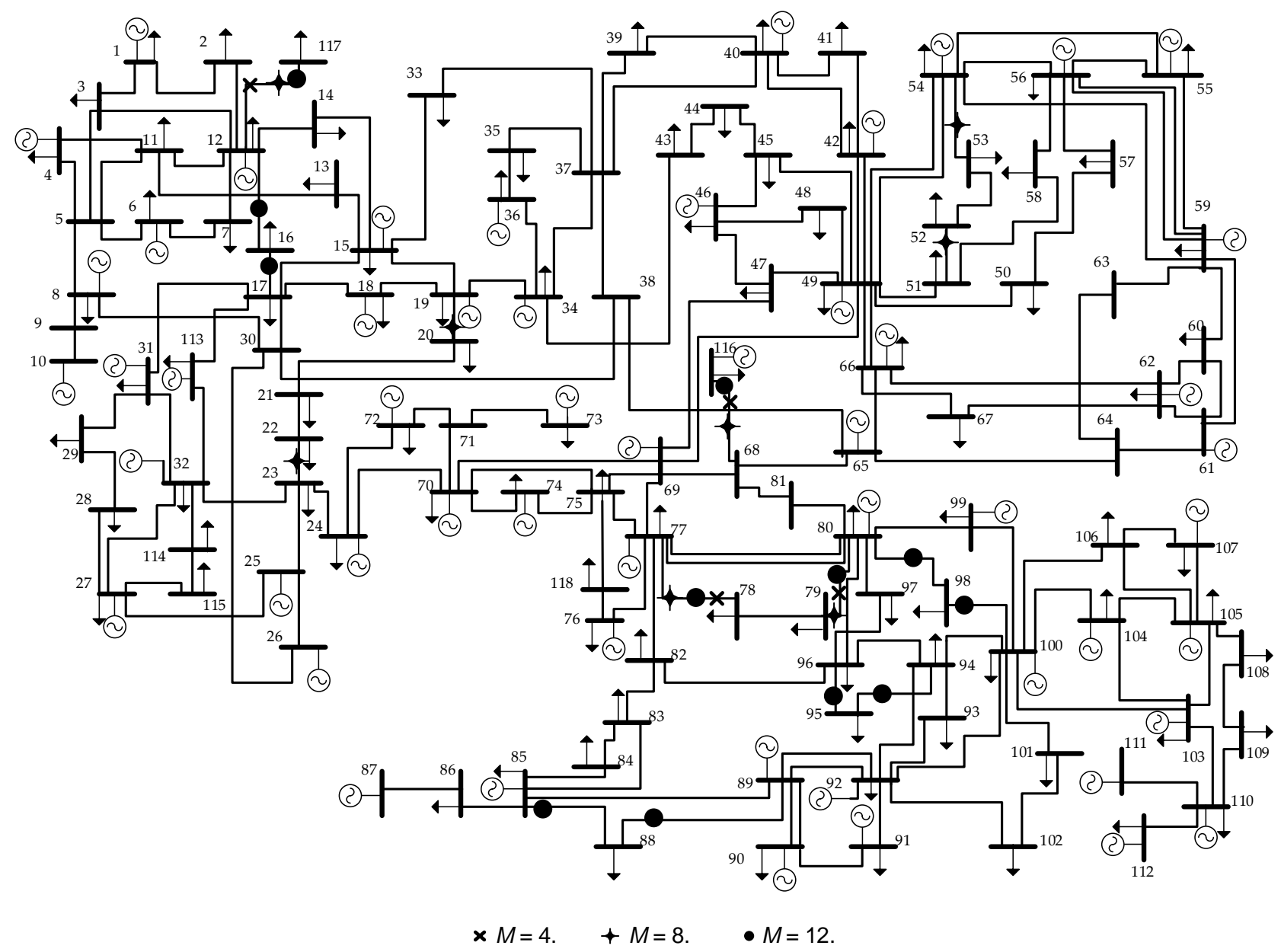

Fig.12: Mejores planes de ataque hallados con las distintas técnicas de búsqueda - Sistema IEEE 118 barras

\section{CONCLUSIONES}

En este artículo se presentó un estudio comparativo de técnicas de solución metaheurísticas aplicadas al problema de interdicción en sistemas de potencia. Los métodos estudiados son BLI, GRASP y AG. La solución del problema de interdicción permite encontrar los elementos más críticos de un sistema de potencia en términos del deslastre de carga que se pueda ocasionar si estos son atacados de forma simultánea.

Las pruebas realizadas los sistemas IEEE de 24 y 118 barras evidencian la aplicabilidad de las técnicas como también sus fortalezas y debilidades. Se encontró que las técnicas BLI y GRASP pueden encontrar soluciones de mejor calidad que el AG. En particular se resalta el desempeño de la BLI en cuanto a la relación de calidad de respuestas vs tiempo de cómputo. La información obtenida con las técnicas bajo estudio (conjunto de contingencias críticas) puede ser utilizada operadores de red para tomar decisiones en la operación que conduzcan a minimizar el impacto de posibles contingencias. Esta información también puede ser utilizada por el planeador para identificar posibles refuerzos en la red o nodos donde sea necesario tener más generación disponible. En un trabajo futuro se tendrán en cuenta otras técnicas de optimización, además de refinamientos al modelo como la inclusión del efecto de tiempos de reposición de activos, fallas en cascada y respuesta de la demanda.

\section{AGRADECIMIENTOS}

Los autores agradecen a la Universidad de Antioquia (UdeA) por el apoyo del proyecto "Sostenibilidad 20162017" para el desarrollo de este trabajo.

\section{REFERENCIAS}

Agudelo, L.; J.M. López-Lezama y N. Muñoz-Galeano, Análisis de vulnerabilidad de sistemas de potencia mediante programación binivel; Revista Información Tecnológica, 25(3), 104-115 (2014)

Alguacil, N.; A. Delgadillo y J. Arroyo, A trilevel programming approach for electric grid defense planning; Computers and Operations Research, 41(1), 282-290 (2014) 
Arroyo, J., Bilevel programming applied to power system vulnerability analysis under multiple contingencies, IET Generation, Transmission \& Distribution, 4 (2), 178 - 190 (2010)

Arroyo, J. y F. Galiana, On the Solution of the Bilevel Programming Formulation of the Terrorist Threat Problem; IEEE Trans. Power Syst., 20 (2), 789 - 797 (2005)

Arroyo, J. y J. Fernández, A Genetic Algorithm Approach for the Analysis of Electric Grid Interdiction with Line Switching, 15th International conference on Intelligent System Applications to Power Systems (ISAP), 1 - 6 (2009)

Correa, J. y J.M. Yusta, Structural vulnerability in transmission systems: cases of Colombia and Spain; Energy Conversion and Management, 77(1), 408-418 (2014)

Cortina, J., Análisis de vulnerabilidad de sistemas de potencia usando técnicas de optimización metaheurística, Tesis de Maestría, Universidad de Antioquia, Facultad de Ingeniería (2016)

Grigg, C.; P. Wong y otros diez y seis autores, The IEEE Reliability Test System - 1996, A report prepared by the Reliability Test System Task Force of the Application of Probability Methods Subcommittee; IEEE Trans. on Power Systems, 14 (3), 1010 - 1020 (1999)

Hong, L.; L. Zhang y Y. Jiao, Discrete differential evolution algorithm for integer linear bilevel programming problems; Journal of Systems Engineering and Electronics, 27(4), 912-919 (2016)

Hong, L.; L. Zhang y Y. Jiao, Solution for integer bilevel programming problems using orthogonal genetic algorithm; Journal of Systems Engineering and Electronics, 25(3), 443-451 (2014)

Kalyanmoy D. y S. Ankur, An efficient and accurate solution methodology for bilevel multi-objecttive programming problems using a hybrid evolutionary-local-search algorithm; Evolutionary Computation, 18(3), 403-449 (2010)

Li, X.; S. Ma y Wang, Y., Multi-population based ensemble mutation method for single objective bilevel optimization problem; IEEE Access. 4, 7262-7274 (2016)

López-Lezama, J.M.; J. Cortina y N. Muñoz-Galeano, Assessment of the electric grid interdiction problem using a nonlinear modeling approach; Electric Power Systems Research, 144, 243-254 (2017)

Salmeron, J. y K. Wood, The value of recovery transformers in protecting an electric transmission grid against attack; IEEE Trans. on Power Systems, 30 (5), 2396 - 2403 (2015)

Salmeron, J.; K. Wood y R. Baldick, Analysis of electric grid security under terrorist threat; IEEE Trans. on Power Systems, 19 (2), $905-912(2004)$

Sayyadipour, S.; G.R. Yousefi y M.A. Latify, Mid-term vulnerability analysis of power systems under international attacks; IET Gener. Transm. Distrib., 10(15), 3745-3755 (2016)

University of Washington Electrical Engineering, Power Systems Test Case, Available online: https://goo.gl/KnfMsf, Último Acceso: 02 de enero de 2018, Rich Christie - May (1993)

Wang, Y. y R. Baldick, Interdiction analysis of electric grids combining cascading outage and medium-term impacts; IEEE Trans. Power Syst. 29(5), 2160-2168 (2014)

Zimmerman, R.; C. Murillo y R. Thomas, Steady-State Operations, Planning, and Analysis Tools for Power Systems Research and Education, IEEE Trans. on Power Systems, 26 (1), 12-19 (2011) 